\title{
The Use Of Statins In Liver Disease: Risk Versus Benefit
}

Dina Halegoua-De Marzio, MD

\section{Introduction}

The National Cholesterol Education Program periodically produces Adult Treatment Panel (ATP) updates as warranted by current clinical scientific research in cholesterol management. ${ }^{1}$ Each of the guideline reports, ATP I, II, and III, has a major role in guiding physicians how to treat abnormal cholesterol levels. Recent clinical trials demonstrate that LDL-lowering therapy with statins (HMG-CoA inhibitors) reduces total mortality, coronary mortality, major coronary events, coronary procedures and strokes in patients with established coronary heart disease. ${ }^{1}$ However, the ATP III has cited active or chronic liver disease as an absolute contraindication to the use of statins. The reason for this contraindication goes back to the initial clinical trials of Lipitor (atorvastatin), which demonstrated that persistent elevations ( $>3$ times the upper limit of normal) in serum transaminases occurred in $0.7 \%$ of patients who received atorvastatin. The incidence of these abnormalities was $0.2 \%, 0.2 \%, 0.6 \%$ and $2.3 \%$ for $10,20,40$ and $80 \mathrm{mg}$ atorvastatin, respectively. ${ }^{2}$ Based on this information, the recommendation held that liver function tests should be performed before the initiation of treatment with statins, following each dosage increase, and periodically thereafter.

Although several reports of significant liver injury associated with statins have appeared in the literature, in reality it occurs rarely. Current labeling of statins is ambiguous; for example, what exactly does active liver disease or persistent unexplained liver function tests (LFTs) elevations mean? Also there is an assumption that acute hepatotoxicity that develops in a patient with impaired liver function would potentially be more fatal, although evidence-based data to support this theory are lacking. The fact remains that statins are the most efficacious drugs for decreasing low-density lipoprotein cholesterol levels, and cardiovascular disease is the leading cause of mortality in the United States. Thus, it is important to establish whether statin therapy should be withheld from patients with liver disease.

\section{Risk of Statin Hepatotoxicity}

The following question must be addressed: are patients with elevated liver enzymes at a higher risk for statin hepatotoxicity? Chalasani et al and Vuppalanchi et al evaluated the affect of starting statin therapy on the LFTs among patients with baseline normal LFTs and those with baseline abnormal LFTs. They determined that patients with baseline LFT elevation had a greater incidence of a mild to moderate increase in LFTs during treatment as compared to those without baseline LFT abnormalities. The study also compared patients on statin therapy with baseline elevated LFTs to patients with elevated baseline LFTs and no history of statin use. The study showed elevations in LFTs regardless of statin use. Therefore, the study surmised that elevations in LFTs in patients with baseline abnormalities while using statins may be due to underlying liver disease and not statin use. ${ }^{3,4}$
One of the most common causes of undiagnosed abnormal LFTs is nonalcoholic steatohepatitis (NASH). NASH is characterized by steatosis, inflammation and occasionally fibrosis of the liver that can progress to cirrhosis. Rallidis et al studied the safety and efficacy of pravastatin treatment on histological markers of disease activity in patients with NASH. The study suggested a possible beneficial role of statins in the treatment of NASH and showed that the patients with NASH were not at an increased risk of LFT abnormities secondary to statin use. ${ }^{5}$ This clarification is important because many patients with NASH are at a higher risk for cardiac events due to common co-morbidities such as metabolic syndrome, hypercholesterolemia, obesity and diabetes.

In clinical practice, the treatment of hyperlipidemia may necessitate an increase in statin dosage to achieve the target goals recommended by the ATP III. This leads to increased concerns about statin-induced hepatotoxicity in patients with chronic liver disease who require high doses of statin. This concern stems from older clinical trials which showed an increased risk of LFT elevations in patients taking high dose statins. ${ }^{2}$ A recent study by Lewis et al compared pravastatin $80 \mathrm{mg}$ to a placebo in a doubleblind clinical trial. The medications were administered daily to hypercholesterolemic subjects greater than 18 years of age with at least a six-month history of compensated chronic liver disease and a low-density lipoprotein cholesterol (LDL-C) level greater than or equal to $100 \mathrm{mg} / \mathrm{dL}$. In comparison to placebo, highdose pravastatin $(80 \mathrm{mg} /$ day $)$ significantly lowered LDL, total cholesterol, and triglycerides and was safe and well tolerated. ${ }^{6}$

Recent research also contests the need for for routine transaminase screening in patients taking statins. In a study by Smith et al, 1002 patients on statins were tested, and $10(1 \%)$ of the patients were identified as having significant LFT elevations while $5(0.5 \%)$ patients were identified as having moderate LFT elevations. None of the elevations appeared to be directly related to statin use, and no adverse outcomes were related to these abnormal lab values. ${ }^{7}$ Therefore, the risk of severe transaminase elevations with statin use is low. In addition, routine transaminase monitoring may be unnecessary since it has low yield and excessive cost. The monitoring may also lead to unnecessary evaluation of slightly abnormal laboratory findings as well as the discontinuation of a highly effective medication.

\section{The Liver Expert Panel and Final Conclusions}

The National Lipid Association (NLA) is a nonprofit, multidisciplinary medical society focused on enhancing the practice of lipid management in clinical medicine. The Task Force, created in June of 2005, was a two-year independent NLA initiative to extensively review and evaluate the safety of statins and other lipid-modifying drugs. ${ }^{8}$ After compilation of the analysis and commissioned research, these experts presented their conclusions to four Expert Panels who reviewed and considered 
the evidence relative to their respective fields of expertise: nephrology, hepatology and neurology.

The Liver Expert Panel published their findings in the American Journal of Cardiology in 2006. The panel's key message was that there is no proof that statins cause life-threatening liver damage. LFT elevations are usually reversible after stopping the statin therapy and do not cause long-term damage. Routine liver enzyme monitoring is not needed during statin therapy. If an isolated asymptomatic transaminase level of one to three times the upper limits of normal is detected, the statin does not need to be discontinued. If the transaminase level is more than three times the upper limits of normal during routine evaluation, the test should be repeated; if it is still elevated, further evaluation should be pursued. Although routine transaminase monitoring in patients taking statins is unnecessary, clinicians should be alert to patient reports of jaundice, malaise, fatigue and other possible signs of hepatotoxicity. If there is an objective evidence of significant liver injury, the statin should be discontinued. In conclusion, statin therapy can be safely administered to patients with chronic liver disease, compensated cirrhosis, and NASH. ${ }^{8}$

\section{References}

1. National Heart, Lung, and Blood Institute. Third report of the National Cholesterol Education Program (NCEP) Expert Panel on Detection, Evaluation, and Treatment of High Blood Cholesterol in Adults (Adult Treatment Panel III). Bethesda, Md: US Department of Health and Human Services, Public Health Service; 2001.

2. “Lipitor Package Insert” Data on file. Pfizer Inc, New York, NY; March 2006 $<$ http://www.lipitor.com/content/index.jsp $>$.

3. Chalasani N, Aljadhey H, Kesterson J, Murray MD, Hall SD. Patient with elevated liver enzymes are not at higher risk for statin hepatotoxicity. Gastroenterology 2004; 126:1287-1292.

4. Vuppalanchi R, Teal E, Chalasani N. Patient with elevated baseline liver enzymes do not have higher frequency of hepatotoxicity from Lovastatin than those with normal baseline liver enzymes. Am J Med Sci 2005; 329:62-65.

5. Rallidis LS, Drakoulis CK, Parasi AS. Pravastatin in patients with nonalcoholic steatohepatitis: results of a pilot study. Atherosclerosis 2004; 174:193-196.

6. Lewis $\mathrm{JH}$, et al. Efficacy and safety of high-dose pravastatin in hypercholesterolemic patients with well-compensated chronic liver disease: Results of a prospective, randomized, double-blind, placebo-controlled, multicenter trial. Hepatology. 2007; 46:1453-63.

7. Smith CG, Bernstein LI, Davis RB, Rind DM, Shmerling RH. Screening for Statin-Related Toxicity: The Yield of Transaminase and Creatine Kinase Measurements in a Primary Care Setting. Arch Intern Med. 2003; 163:688-692.

8. Cohen DE, Anania, FA, Chalasani, N. An Assessment of Statin Safetly by Hepatotogists. Am J Cardiol 2006; 97(suppl): 77c-81c.
Photograph courtesy of Lisa Teng, MD

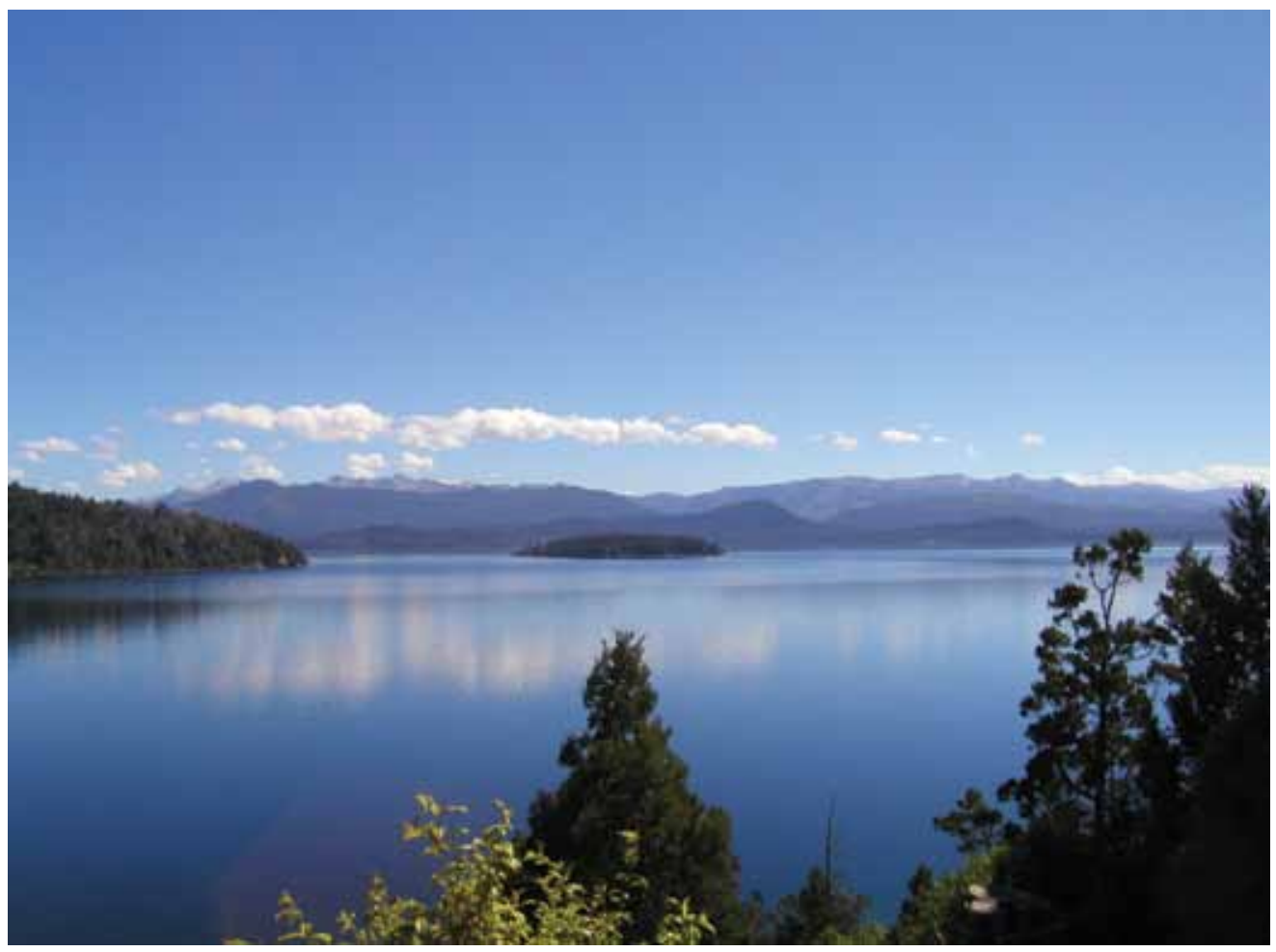

